\title{
Reinforcement and cue factors in reversal learning'
}

\author{
Howard H. Kendler and Joseph Kimm
} UNIVERSITY OF CALIFORNIA, SANTA BARBARA

\begin{abstract}
In a $2 \times 2 \times 2$ experimental design involving learning to a criterion and overlearning, small and large food reward, and a differentiated and undifferentiated maze, 35 rats learned a spatial discrimination and then were reversed. An analysis of the reversal learning data indicated all main effects were significant as well as both double interactions involving the amount of food. No overlearning reversal effect was obtained.
\end{abstract}

\section{Problem}

This study is designed to test an analysis of selective learning that might account for the success of some (e.g. Reid, 1953) and the failure of others (e.g. Erlebacher, 1963), to obtain the overlearning reversal effect (ORE). The analysis is based upon the assumption that after a discrimination habit is learned the $\mathrm{S}$ responds at the choice point to a pattern of stimuli with a fixed number of elements. Under conditions that encourage fractional anticipatory goal responses $\left(\mathrm{r}_{\mathrm{g}}\right.$ 's) at the choice point, the additional assumption is made that overlearning changes the proportion of internal and external cues in the choice-point pattern; initially cues from external sources (the choice point) predominate, but later the proportion of internal cues $\left(\mathrm{s}_{\mathrm{g}}\right.$ 's) resulting from the $r_{g}$ 's increases. The higher the proportion of the $\mathrm{s}_{\mathrm{g}}$ 's in the stimulus pattern at the choice point the more rapidly will reversal learning be executed. This follows from the expectation that the goal responses $\left(R_{G}{ }^{\prime} s\right)$ in the initially correct end-box would be extinguished during the early stages of reversal learning because of the removal of the reinforcement. Through generalization the $\mathrm{r}_{\mathrm{g}}$ 's at the choice point would be weakened with a correlated reduction in the $\mathrm{s}_{\mathrm{g}}$ 's. Because these $\mathrm{s}_{\mathrm{g}}$ 's had been conditioned to the response that is incorrect during reversal learning, their elimination from the stimulus pattern at the choice point should hasten the extinction of the initially learned habit and thereby facilitate reversal learning.

The above analysis was translated into a $2 \times 2 \times 2$ experimental design with the following pairs of variables: a large (L) and small (S) amount of food in the goal box, a differentiated (D) and undifferentiated (U) T-maze, and learning to a criterion $(\mathrm{C})$ and overlearning $(\mathrm{O})$. It was expected that the evocation of $\mathrm{r}_{\mathrm{g}}$ 's at the choice point would be encouraged by the large amount of food, the differentiated maze, and overlearning, and therefore these variables should operate to facilitate reversal learning.

\section{Subjects}

Forty-eight male albino rats, 82 days of age, obtained from Cheek-Jones were randomly assigned to one of eight groups (LCD, SOU, etc.). Of these, 13 had to be discarded because of respiratory disease.
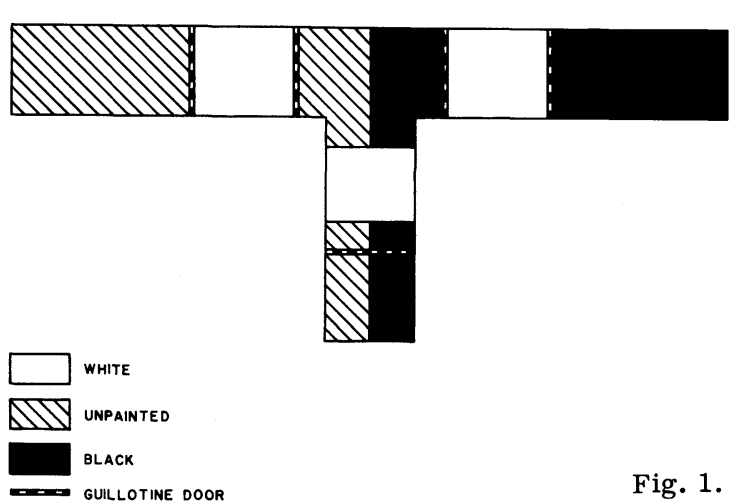

Fig. 1 .

\section{Apparatus}

Two single-unit $\mathrm{T}$-mazes of identical dimensions were used. A scaled floor plan of the differentiated maze appears in Fig. 1. The floors, walls, and doors of the unpainted section were covered with a fine wire mesh. A 6-w light bulb was placed over the black goal box. The top of the differentiated maze was covered with hardware cloth, while a translucent plastic sheet covered the undifferentiated maze. The entire undifferentiated maze was painted a gray and was illuminated evenly by overhead lights.

\section{Procedure}

After preliminary handling, nonrewarded exploratory experience in the maze, and tests for position preference, training began. Five trials daily were given to each $\mathrm{S}$ during the initial two days of training; thereafter the Ss received 10 trials daily. At least $15 \mathrm{~min}$. intervened between successive trials for each S. Although the amount of food in the goal boxes differed for $S$ (two 45-mg pellets) and L (four Purina lab checkers) Ss, it was planned that Ss would consume approximately the same amount. The eating time of all Ss was limited to $8 \mathrm{sec}$, the time required by the $\mathrm{S}$ Ss to consume the two pellets during the early stages of training. On incorrect trials, Ss were confined in the end-box for $8 \mathrm{sec}$. The criterion of initial learning was 18 correct trials on two successive days ( 20 trials) with no more than one error committed on the last day. The Ss in the $\mathrm{C}$ groups were reversed the day after they reached criterion while the $O$ Ss received 150 additional training trials. The criterion of reversal learning was 10 successive correct responses.

The Ss received daily periods of $1-1 / 2 \mathrm{hr}$. ad lib feeding beginning no sooner than $15 \mathrm{~min}$. after the completion of their training trials.

\section{Results and Diseussion}

An analysis of variance based on unequal sample sizes revealed no significant effects for initial learning, although each group with the differentiated maze learned 
Table 1. Trials to Criterion and Perseverative Errors During Reversal Learning

\begin{tabular}{lllllll} 
& \multicolumn{6}{c}{ Differentiated Maze } \\
\cline { 5 - 6 } Group & $\underline{N}$ & Mdn. & $\underline{M}$ & $\underline{\text { SD }}$ & $\underline{\text { P-Errors }}$ \\
SCD & 5 & 11.0 & 11.0 & 1.7 & 3.0 \\
SOD & 5 & 17.0 & 18.4 & 4.4 & 7.6 \\
ICD & 5 & 3.0 & 5.8 & 4.8 & 3.0 \\
LOD & 4 & 5.0 & 6.5 & 3.8 & 3.3
\end{tabular}

more rapidly than did the comparable groups with the undifferentiated maze. (The mean number of trials ranged from 24 to 40 .)

Table 1 reports the results of reversal learning, while Table 2 indicates the results of analysis of variance. The Duncan multiple range tests for unequal sample sizes (Kramer, 1956) resulted in significant differences between Groups SCU and SOU ( $p<.001)$, SOU and LOU $(\mathrm{p}<.001)$, and SOU and SOD $(\mathrm{p}<.05)$.

In general the results fail to offer unqualified support to the theory that initiated this study, mainly because of the absence of any ORE. Nevertheless the theoretical ideas do receive some support from the fact that the experimental variables investigated influenced the speed of reversal learning. What seems to be the case is that overlearning with a small reward retards reversal learning but overlearning with a large reward does not. In addition, a differentiated maze, which presumably encourages the evocation of $r_{g}$ 's at the choice point and start box, facilitates reversal learning as compared to an undifferentiated maze, with a small reward, but not with a large one.

In interpreting the influence of the size of reward on reversal learning two factors must be considered. First, the experiment was designed so that the time allowed for eating would be the same for both S and L Ss. But near the end of the experiment the SSs were consuming their pellets more rapidly than at the beginning. Assuming that the eating rate of the L Ss also increased, it is likely that they consumed more food and ate longer than did the S Ss. Second, the separate influences of the size of the goal object during initial and reversal learning are not isolated by the present experimental design. According to the $r_{g}$ formulation that suggested this study, a large goal object should facilitate reversal learning because it would cause more rapid extinction of the initially learned habit. An analysis of the perseverative errors (see Table 2), the successive errors before the first correct response during reversal learning, barely failed to yield a significant amount-of-reward effect; $F(1,27)=4.07, .05<p<.10$. Hulse's (1958) finding that with a continuous reinforcement schedule a large food reward (1.0 gr) produced faster extinction than did a small reward (.08 gr), would suggest that additional information should be gathered about the role of the size of the reward during initial learning on the speed of reversal learning.

\begin{tabular}{|c|c|c|c|c|c|}
\hline \multirow[b]{2}{*}{ Group } & \multirow[b]{2}{*}{$\underline{N}$} & \multicolumn{4}{|c|}{ Undifferentiated Maze } \\
\hline & & Mdn. & M & $\underline{\mathrm{SD}}$ & $\stackrel{\frac{M}{P}}{\text { P-Errors }}$ \\
\hline $\mathrm{SCU}$ & 4 & 11.5 & $17 \cdot 5$ & 12.5 & 4.8 \\
\hline SOU & 4 & 47.0 & 48.3 & 18.4 & $9 \cdot 3$ \\
\hline LCU & 5 & $9 \cdot 0$ & 8.0 & 3.9 & 1.5 \\
\hline LOU & 3 & 8.0 & $7 \cdot 3$ & 3.3 & 1.7 \\
\hline
\end{tabular}

Table 2. Analysis of Variance of Number of Trials to Reach Criterion of Reversal Learning

$\begin{array}{lccc}\text { Source } & \text { df } & \underline{M S} & \underline{F} \\ \text { Overlearning (0) } & 1 & 774.14 & 8.81 * * \\ \text { Amount Food (A) } & 1 & 2420.44 & 27.53^{* * *} \\ \text { Maze (M) } & 1 & 823.56 & 9.37^{* *} \\ \text { OxA } & 1 & 771.44 & 8.78 * * \\ \text { OxM } & 1 & 256.60 & 2.92 \\ \text { AxM } & 1 & 588.38 & 6.70 * \\ \text { OxAxM } & 1 & 324.28 & 3.69 \\ \text { Error } & 27 & 87.90 & -\ldots \\ * \mathrm{p}<.05 & & & \\ * * \mathrm{p}<.01 & & & \\ * * * \mathrm{p}<.001 & & \end{array}$

\section{References}

ERLEBACHER, ALBERT. Reversal learning in rats as a function of percentage of reinforcement and degree of learning. J. exp. Psychol., 1963, 66, 84-90.

HULSE, STEWART H., JR. Amount and percentage of reinforcement and duration of goal confinement in conditioning and extinction. J.exp. Psychol., 1958, 56, 48-56.

KRAMER, C. Y. Extension of multiple range tests to group means with unequal number of replications. Biometrics, 1956, 12, 307-310.

REID, LYNE STARLING. The development of noncontinuity behavior through continuity learning. J. exp. Psychol., 1953, 46, 107-112.

\section{Note}

1. This research was supported by the Office of Naval Research. The authors are indebted to Mr. Michael R. Meeker for his design of the computer programs used to analyze the data, and to Dr. Tracy S. Kendler for her helpful reading of the manuscript. 